TRANSACTIONS OF THE

AMERICAN MATHEMATICAL SOCIETY

Volume 360, Number 8, August 2008, Pages 4169-4188

S 0002-9947(08)04488-7

Article electronically published on March 11, 2008

\title{
COHOMOLOGY OF AFFINE ARTIN GROUPS AND APPLICATIONS
}

\author{
FILIPPO CALLEGARO, DAVIDE MORONI, AND MARIO SALVETTI
}

\begin{abstract}
The result of this paper is the determination of the cohomology of Artin groups of type $A_{n}, B_{n}$ and $\tilde{A}_{n}$ with non-trivial local coefficients. The main result is an explicit computation of the cohomology of the Artin group of type $B_{n}$ with coefficients over the module $\mathbb{Q}\left[q^{ \pm 1}, t^{ \pm 1}\right]$. Here the first $n-1$ standard generators of the group act by $(-q)$-multiplication, while the last one acts by $(-t)$-multiplication. The proof uses some technical results from previous papers plus computations over a suitable spectral sequence. The remaining cases follow from an application of Shapiro's lemma, by considering some well-known inclusions: we obtain the rational cohomology of the Artin group of affine type $\tilde{A}_{n}$ as well as the cohomology of the classical braid group $\mathrm{Br}_{n}$ with coefficients in the $n$-dimensional representation presented in Tong, Yang, and $\mathrm{Ma}$ (1996). The topological counterpart is the explicit construction of finite $\mathrm{CW}$-complexes endowed with a free action of the Artin groups, which are known to be $K(\pi, 1)$ spaces in some cases (including finite type groups). Particularly simple formulas for the Euler-characteristic of these orbit spaces are derived.
\end{abstract}

\section{INTRODUCTION}

Recall that for each Coxeter group $W$ one has a group extension $G_{W}$, usually called an Artin group of type $W$ (see Section 2). In this paper we give a detailed calculation of the cohomology of some Artin groups with non-trivial local coefficients. Let $R:=\mathbb{Q}\left[q^{ \pm 1}, t^{ \pm 1}\right]$ be the ring of two-parameter Laurent polynomials. The main result (Theorem 1.1) is the computation of the cohomology of the Artin group $G_{B_{n}}$ (of type $B_{n}$ ) with coefficients in the module $R_{q, t}$. The latter is the ring $R$ with the module structure defined as follows: the generators associated with the first $n-1$ nodes of the Dynkin diagram of $B_{n}$ act by $(-q)$-multiplication; the one associated to the last node acts by $(-t)$-multiplication.

Let $\varphi_{m}(q)$ be the $m$-th cyclotomic polynomial in the variable $q$. Define the $R$-modules $(m>1, i \geq 0)$

$$
\{m\}_{i}=R /\left(\varphi_{m}(q), q^{i} t+1\right)
$$

and for $m=1$, set

$$
\{1\}_{i}=R /\left(q^{i} t+1\right)
$$

Notice that the modules $\{m\}_{i}$ are all non-isomorphic as $R$-modules. $\{m\}_{i}$ and $\left\{m^{\prime}\right\}_{i^{\prime}}$ are isomorphic as $\mathbb{Q}\left[q^{ \pm 1}\right]$-modules if and only if $m=m^{\prime}$ and are isomorphic

Received by the editors June 20, 2006.

2000 Mathematics Subject Classification. Primary 20J06, 20F36.

Key words and phrases. Affine Artin groups, twisted cohomology, group representations.

The third author is partially supported by M.U.R.S.T. $40 \%$.

(c)2008 American Mathematical Society Reverts to public domain 28 years from publication 4169 
as $\mathbb{Q}\left[t^{ \pm 1}\right]$-modules if and only if $\phi(m)=\phi\left(m^{\prime}\right)\left(\phi\right.$ is the Euler function) and $\frac{m}{(m, i)}=$ $\frac{m^{\prime}}{\left(m, i^{\prime}\right)}$.

Our main result is the following.

Theorem 1.1.

$$
H^{i}\left(G_{B_{n}}, R_{q, t}\right)= \begin{cases}\bigoplus_{d \mid n, 0 \leq k \leq d-2}\{d\}_{k} \oplus\{1\}_{n-1} & \text { if } i=n, \\ \bigoplus_{d \mid n, 0 \leq k \leq d-2, d \leq \frac{n}{j+1}}\{d\}_{k} & \text { if } i=n-2 j, \\ \bigoplus_{d \nmid n, d \leq \frac{n}{j+1}}\{d\}_{n-1} & \text { if } i=n-2 j-1 .\end{cases}
$$

The proof uses the spectral sequence associated with a natural filtration of the algebraic complex exhibited in Sal94, plus some technical results from DCPS01.

We apply Shapiro's lemma to a well-known inclusion of $G_{\tilde{A}_{n-1}}$ into $G_{B_{n}}$ to derive the cohomology of $G_{\tilde{A}_{n-1}}$ over the module $\mathbb{Q}\left[q^{ \pm 1}\right]$, the action of each standard generator being $(-q)$-multiplication.

By considering another natural inclusion of $G_{B_{n}}$ into the classical braid group $\mathrm{Br}_{n+1}:=G_{A_{n}}$, we also use Shapiro's lemma in order to identify the cohomology of $G_{B_{n}}$ with coefficients in $R_{q, t}$ with that of $\mathrm{Br}_{n+1}$ with coefficients in the irreducible $(n+1)$-dimensional representation of $\mathrm{Br}_{n+1}$ found in TYM96, twisted by an abelian representation. We derive the trivial $\mathbb{Q}$-cohomology of $G_{\tilde{A}_{n-1}}$ as well as the cohomology of the braid group over the irreducible representation in TYM96.

Computation of the cohomology of Artin groups was done by several people: for classical braid groups and trivial coefficients it was first given by F. Cohen Coh76, and independently by A. Vaŭnštel̆n Vaü78] (see also [Arn68, Bri71, BS72, Fuk70]). For Artin groups of type $C_{n}, D_{n}$, see Gor78, while for the exceptional cases, see [Sal94], where the $\mathbb{Z}$-module structure was given, while the ring structure was computed in Lan00. The case of non-trivial coefficients over the module of Laurent polynomials $\mathbb{Q}\left[q^{ \pm 1}\right]$ is interesting because of its relation with the trivial $\mathbb{Q}$ cohomology of the Milnor fibre of the naturally associated bundle. For the classical braid groups, see Fre88, Mar96, DCPS01, while for the cases $C_{n}, D_{n}$, see DCPSS99]. For computations over the integral Laurent polynomials $\mathbb{Z}\left[q^{ \pm 1}\right]$, see CS98 for the exceptional cases and recently [Cal06] for the case of braid groups, and [DCSS97] for the top cohomologies in all cases. In the case of Artin groups of non-finite type, some computations were given in [SS97] and [CD95].

The computations of Theorem 1.1 could be partially extended to integral coefficients; however, major complications occur because the Laurent polynomial ring $\mathbb{Z}\left[q^{ \pm 1}\right]$ is not a P.I.D.

In the last part we also indicate (see [CMS]) an explicit construction of finite $\mathrm{CW}$-complexes which are retracts of orbit spaces associated to Artin groups. The construction works as in SSal94, with few variations necessary for infinite type Artin groups (see also CD95 for a different construction). The Artin group identifies with the fundamental group of the orbit space, and the standard presentation follows easily (see Bri71, Dũn83, vdL83]). The Euler characteristic of the orbit space reduces to that of a simplicial complex and in some cases one has a particularly simple formula. It is conjectured that such orbit spaces are always $K(\pi, 1)$ spaces; for the affine groups, this is known in the cases $\tilde{A}_{n}, \tilde{C}_{n}$ (see Oko79, CP03) and recently also for $\tilde{B}_{n}$ ([CMS1]) (see also CD95 for a different class of Artin groups of infinite type). 
Notice also the geometrical meaning of the two-parameter cohomology of $G_{B_{n}}$ : similar to the one-parameter case, it is equivalent to the trivial cohomology of the "homotopy-Milnor fibre" associated with the natural map of the orbit space onto a two-dimensional torus.

The main results of this paper were announced (without proof) in CMS.

\section{Preliminary Results}

In this section we briefly fix the notation and recall some preliminary results.

2.1. Coxeter groups and Artin groups. A Coxeter graph is a finite undirected graph, whose edges are labelled with integers $\geq 3$ or with the symbol $\infty$.

Let $S$ be the vertex set of a Coxeter graph. For every pair of vertices $s, t \in S$ $(s \neq t)$ joined by an edge, define $m(s, t)$ to be the label of the edge joining them. If $s, t$ are not joined by an edge, set by convention $m(s, t)=2$. Also let $m(s, s)=1$ (see [Bou68, Hum90]).

Two groups are associated with a Coxeter graph: the Coxeter group $W$ defined by

$$
\left.W=\langle s \in S|(s t)^{m(s, t)}=1 \forall s, t \in S \text { such that } m(s, t) \neq \infty\right\rangle
$$

and the Artin group $G$ defined by (see BS72]):

$$
G=\langle s \in S| \underbrace{s t s t \ldots}_{m(s, t) \text {-terms }}=\underbrace{t s t s \ldots}_{m(s, t) \text {-terms }} \forall s, t \in S \text { such that } m(s, t) \neq \infty\rangle .
$$

Loosely speaking, $G$ is the group obtained by dropping the relations $s^{2}=1(s \in S)$ in the presentation for $W$.

In this paper, we are primarily interested in Artin groups associated with Coxeter graphs of types $A_{n}, B_{n}$ and $\tilde{A}_{n-1}$ (see Figure प).
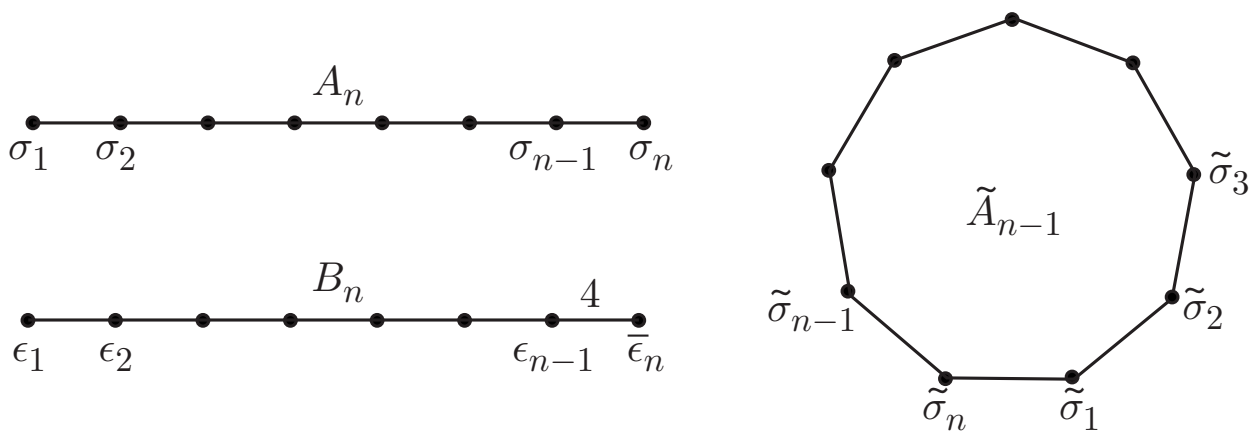

Figure 1. Coxeter graphs of types $A_{n}, B_{n}(n \geq 2)$ and $\tilde{A}_{n-1}$ $(n \geq 3)$. Labels equal to 3 , as usual, are not shown. Moreover, to fix notation, every vertex is labelled with the corresponding generator in the Artin group. 
2.2. Inclusions of Artin groups. Let $\mathrm{Br}_{n+1}:=G_{A_{n}}$ be the braid group on $n+1$ strands and $\mathrm{Br}_{n+1}^{n+1}<\mathrm{Br}_{n+1}$ be the subgroup of braids fixing the $(n+1)$-st strand. The group $\mathrm{Br}_{n+1}^{n+1}$ is called the annular braid group. Let $K_{n+1}=\left\{p_{1}, \ldots, p_{n+1}\right\}$ be a set of $n+1$ distinct points in $\mathbb{C}$. The classical braid group $\operatorname{Br}_{n+1}=G_{A_{n}}$ can be realized as the fundamental group of the space of unordered configurations of $n+1$ points in $\mathbb{C}$ with basepoint $K_{n+1}$ (see the left part of Figure 2, with $\left.K_{6}=\{1, \ldots, 6\}\right)$. We can now think of the subgroup $\mathrm{Br}_{n+1}^{n+1}<\mathrm{Br}_{n+1}$ as the fundamental group of the space of unordered configurations of $n$ points in $\mathbb{C}^{*}$ : in fact if we take $p_{n+1}=0$ and $p_{i} \in S^{1} \subset \mathbb{C}$ for $i \in 1, \ldots, n$, since for a braid $\beta \in \operatorname{Br}_{n+1}^{n+1}$ the orbit of the $(n+1)$-st point can be considered constant, up to homotopy, we can think of $\beta$ as a braid with $n$ strands in the annulus (see the right part of Figure 21).

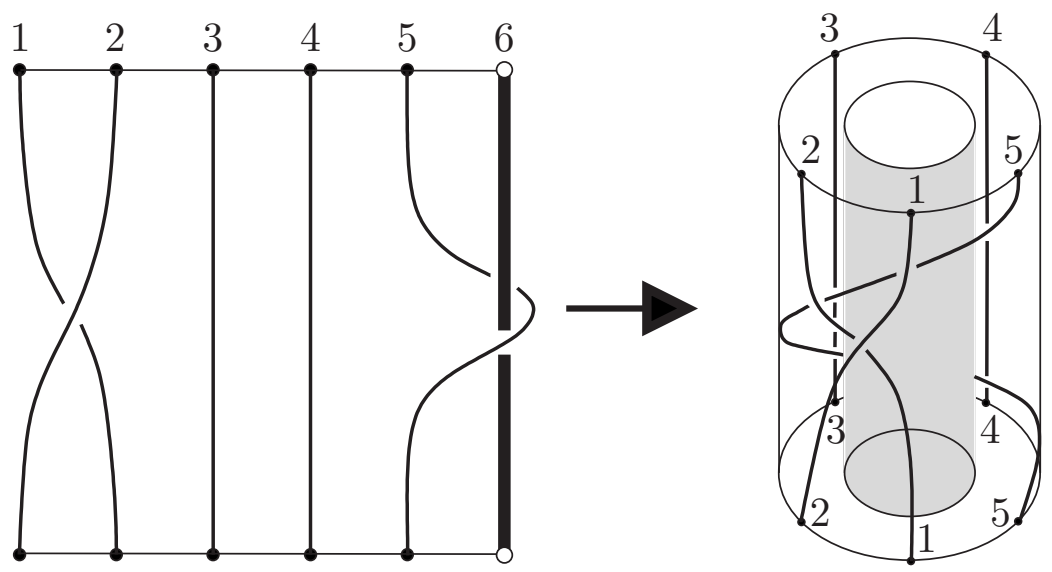

Figure 2. A braid in $\mathrm{Br}_{6}^{6}$ represented as an annular braid on 5 strands

It is well known that the annular braid group is isomorphic to the Artin group $G_{B_{n}}$ of type $B_{n}$. For a proof of the following theorem, see [Cri99] or [Lam94].

Theorem 2.1. Let $\sigma_{1}, \ldots, \sigma_{n}$ and $\epsilon_{1}, \ldots, \epsilon_{n-1}, \bar{\epsilon}_{n}$ be respectively the standard generators for $G_{A_{n}}$ and $G_{B_{n}}$. Then, the map

$$
\begin{aligned}
G_{B_{n}} & \rightarrow \mathrm{Br}_{n+1}^{n+1}<\mathrm{Br}_{n+1}, \\
\epsilon_{i} & \mapsto \sigma_{i} \quad \text { for } 1 \leq i \leq n-1, \\
\bar{\epsilon}_{n} & \mapsto \sigma_{n}^{2}
\end{aligned}
$$

is an isomorphism.

Using the suggestion given by the identification with the annular braid group, a new interesting presentation for $G_{B_{n}}$ can be worked out. Let $\tau=\bar{\epsilon}_{n} \epsilon_{n-1} \cdots \epsilon_{2} \epsilon_{1}$. It is easy to verify that

$$
\tau^{-1} \epsilon_{i} \tau=\epsilon_{i+1} \quad \text { for } 1 \leq i<n-1 ;
$$

i.e., conjugation by $\tau$ shifts forward the first $n-2$ standard generators. By analogy, let $\epsilon_{n}=\tau^{-1} \epsilon_{n-1} \tau$. We have the following. 


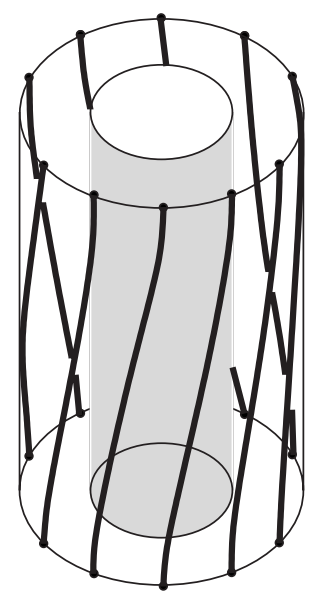

Figure 3. As an annular braid the element $\tau$ is obtained by turning the bottom annulus by a rotation of $2 \pi / n$.

Theorem 2.2 ([KP02]). The group $G_{B_{n}}$ has presentation $\langle\mathcal{G} \mid \mathcal{R}\rangle$ where

$$
\begin{aligned}
\mathcal{G} & =\left\{\tau, \epsilon_{1}, \epsilon_{2}, \ldots, \epsilon_{n}\right\}, \\
\mathcal{R} & =\left\{\epsilon_{i} \epsilon_{j}=\epsilon_{j} \epsilon_{i} \quad \text { for } i \neq j-1, j+1\right\} \cup \\
& \left\{\epsilon_{i} \epsilon_{i+1} \epsilon_{i}=\epsilon_{i+1} \epsilon_{i} \epsilon_{i+1}\right\} \cup \\
& \left\{\tau^{-1} \epsilon_{i} \tau=\epsilon_{i+1}\right\}
\end{aligned}
$$

where all indexes should be considered modulo $n$.

Letting $\tilde{\sigma}_{1}, \tilde{\sigma}_{2}, \ldots, \tilde{\sigma}_{n}$ be the standard generators of the Artin group of type $\tilde{A}_{n-1}$, we have the following straightforward corollary:

Corollary 2.3 (KP02). The map

$$
G_{\tilde{A}_{n-1}} \ni \tilde{\sigma}_{i} \mapsto \epsilon_{i} \in G_{B_{n}}
$$

gives an isomorphism between the group $G_{\tilde{A}_{n-1}}$ and the subgroup of $G_{B_{n}}$ generated by $\epsilon_{1}, \ldots, \epsilon_{n}$. Moreover, we have a semidirect product decomposition $G_{B_{n}} \cong$ $G_{\tilde{A}_{n-1}} \rtimes\langle\tau\rangle$.

We have thus a "curious" inclusion of the Artin group of infinite type $\tilde{A}_{n-1}$ into the Artin group of finite type $B_{n}$.

Remark 2.4. The proof of Theorem 2.2 presented in the original paper is algebraic and based on Tietze moves; a somewhat more concise proof can however be obtained by standard topological constructions. Indeed, one can exhibit an explicit infinite cyclic covering $K\left(G_{\tilde{A}_{n-1}}, 1\right) \rightarrow K\left(G_{B_{n}}, 1\right)$ (see All02).

2.3. (q, t)-weighted Poincaré series for $\mathbf{B}_{\mathbf{n}}$. For future use in cohomology computations, we are interested in a $(q, t)$-analog of the usual Poincaré series for $B_{n}$, that is, an analog of the Poincaré series with coefficients in the ring $R=\mathbb{Q}\left[q^{ \pm 1}, t^{ \pm 1}\right]$ of Laurent polynomials. This result and similar ones are studied in Rei93, to which we refer for details. We also use classical results from Bou68, Hum90 without further reference. 
Consider the Coxeter group $W$ of type $B_{n}$ with its standard generating reflections $s_{1}, s_{2}, \ldots, s_{n}$. For $w \in W$, let $n(w)$ be the number of times $s_{n}$ appears in a reduced expression for $w$. By standard facts, $n(w)$ is well defined.

We define the $(q, t)$-weighted Poincaré series for the Coxeter group of type $B_{n}$ as the sum

$$
W(q, t)=\sum_{w \in W} q^{\ell(w)-n(w)} t^{n(w)}
$$

where $\ell$ is the length function.

We recall some notation. We define the $q$-analog of the number $m$ by the polynomial

$$
[m]_{q}:=1+q+\cdots+q^{m-1}=\frac{q^{m}-1}{q-1} .
$$

Notice that $[m]=\prod_{i \mid m, i \neq 1} \varphi_{m}(q)$, where we denote with $\varphi_{m}(q)$ the $m$-th cyclotomic polynomial in the variable $q$. Moreover we define the $q$-factorial analog $[m]_{q}$ ! as the product

$$
\prod_{i=1}^{m}[i]_{q}
$$

and the $q$-analog of the binomial $\left(\begin{array}{c}m \\ i\end{array}\right)$ as the polynomial

$$
\left[\begin{array}{c}
m \\
i
\end{array}\right]_{q}:=\frac{[m]_{q} !}{[i]_{q} ![m-i]_{q} !}
$$

We can also define the $(q, t)$-analog of an even number

$$
[2 m]_{q, t}:=[m]_{q}\left(1+t q^{m-1}\right)
$$

and of the double factorial

$$
[2 m]_{q, t} ! !:=\prod_{i=1}^{m}[2 i]_{q, t}=[m]_{q} ! \prod_{i=0}^{m-1}\left(1+t q^{i}\right) .
$$

Finally, we define the polynomial

$$
\left[\begin{array}{c}
m \\
i
\end{array}\right]_{q, t}^{\prime}:=\frac{[2 m]_{q, t} ! !}{[2 i]_{q, t} ! ![m-i]_{q} !}=\left[\begin{array}{c}
m \\
i
\end{array}\right]_{q} \prod_{j=i}^{m-1}\left(1+t q^{j}\right) .
$$

Proposition 2.5 (Rei93]).

$$
W(q, t)=[2 n]_{q, t} ! ! .
$$

Proof. Consider the parabolic subgroup $W_{I}$ associated with the subset of reflections $I=\left\{s_{1}, \ldots, s_{n-1}\right\}$. Notice that $W_{I}$ is isomorphic to the symmetric group on $n$ letters $A_{n-1}$ and that it has index $2^{n}$ in $B_{n}$. Let $W^{I}$ be the set of minimal coset representatives for $W / W_{I}$. Then, by multiplicative properties on reduced expressions:

$$
\begin{aligned}
W(q, t) & =\sum_{w \in W} q^{\ell(w)-n(w)} t^{n(w)} \\
& =\left(\sum_{w^{\prime} \in W^{I}} q^{\ell\left(w^{\prime}\right)-n\left(w^{\prime}\right)} t^{n\left(w^{\prime}\right)}\right) \cdot\left(\sum_{w^{\prime \prime} \in W_{I}} q^{\ell\left(w^{\prime \prime}\right)-n\left(w^{\prime \prime}\right)} t^{n\left(w^{\prime \prime}\right)}\right) .
\end{aligned}
$$


Clearly, for elements $w^{\prime \prime} \in W_{I}$, we have $n\left(w^{\prime \prime}\right)=0$; so the second factor in (2.1) reduces to the well-known Poincaré series for $A_{n-1}$ :

$$
\sum_{w^{\prime \prime} \in W_{I}} q^{\ell\left(w^{\prime \prime}\right)-n\left(w^{\prime \prime}\right)} t^{n\left(w^{\prime \prime}\right)}=[n]_{q} !
$$

To deal with the first factor, instead, we explicitly enumerate the elements of $W^{I}$. Let $p_{i}=s_{i} s_{i+1} \cdots s_{n}$ for $1 \leq i \leq n$. Then, it can be easily verified that $W^{I}=$ $\left\{p_{i_{r}} p_{i_{r-1}} \cdots p_{i_{2}} p_{i_{1}} \mid i_{1}<i_{2}<\cdots<i_{r-1}<i_{r}\right\}$. Notice that $n\left(p_{i_{r}} p_{i_{r-1}} \cdots p_{i_{2}} p_{i_{1}}\right)=r$ and $\ell\left(p_{i_{r}} p_{i_{r-1}} \cdots p_{i_{2}} p_{i_{1}}\right)=\sum_{j=1}^{r} \ell\left(p_{i_{j}}\right)=\sum_{j=1}^{r}\left(n+1-i_{j}\right)$. Thus,

$$
\sum_{w^{\prime} \in W^{I}} q^{\ell\left(w^{\prime}\right)-n\left(w^{\prime}\right)} t^{n\left(w^{\prime}\right)}=\prod_{i=0}^{n-1}\left(1+t q^{i}\right) .
$$

Finally,

$$
W(q, t)=\left(\prod_{i=0}^{n-1}\left(1+t q^{i}\right)\right)[n]_{q} !=[2 n]_{q, t} ! !
$$

\section{The cohomology of $G_{B_{n}}$}

3.1. Proof of the main theorem. In this section we prove Theorem 1.1 enunciated in the introduction. We use the notation given in the introduction.

To perform our computation we will use the complex discovered in Sal94, DCS96 (notice: an equivalent complex was discovered by different methods in Squ94), and the spectral sequence induced by a natural filtration.

The complex that computes the cohomology of $G_{B_{n}}$ over $R_{q, t}$ is given as follows (see [Sal94]):

$$
C_{n}^{*}=\bigoplus_{\Gamma \subset I_{n}} R \cdot \Gamma
$$

where $I_{n}$ denote the set $\{1, \ldots, n\}$ and the graduation is given by $|\Gamma|$.

The set $I_{n}$ corresponds to the set of nodes of the Dynkin diagram of $B_{n}$ and in particular the last element, $n$, corresponds to the last node.

It is useful to consider also the complex $\bar{C}_{n}^{*}$ for the cohomology of $G_{A_{n}}$ on the local system $R_{q, t}$. In this case the action associated with a standard generator is always the $(-q)$-multiplication and so the complex $\bar{C}_{n}^{*}$ and its cohomology are free as $\mathbb{Q}\left[t^{ \pm}\right]$-modules. The complex $\bar{C}_{n}^{*}$ is isomorphic to $C_{n}^{*}$ as an $R$-module. In both complexes the coboundary map is

$$
\delta(q, t)(\Gamma)=\sum_{j \in I_{n} \backslash \Gamma}(-1)^{\sigma(j, \Gamma)} \frac{W_{\Gamma \cup\{j\}}(q, t)}{W_{\Gamma}(q, t)}(\Gamma \cup\{j\})
$$

where $\sigma(j, \Gamma)$ is the number of elements of $\Gamma$ that are less than $j$. In the case $A_{n}$, $W_{\Gamma}(q, t)$ is the Poincaré polynomial of the parabolic subgroup $W_{\Gamma} \subset A_{n}$ generated by the elements in the set $\Gamma$, with weight $-q$ for each standard generator, while in the case $B_{n}, W_{\Gamma}(q, t)$ is the Poincaré polynomial of the parabolic subgroup $W_{\Gamma} \subset B_{n}$ generated by the elements in the set $\Gamma$, with weight $-q$ for the first $n-1$ generators and $-t$ for the last generator.

Using Proposition 2.5 we can give an explicit computation of the coefficients $\frac{W_{\Gamma \cup\{j\}}(q, t)}{W_{\Gamma}(q, t)}$. For any $\Gamma \subset I_{n}$, let $\bar{\Gamma}$ be the subgraph of the Dynkin diagram $B_{n}$ 
which is spanned by $\Gamma$. Recall that if $\bar{\Gamma}$ is a connected component of the Dynkin diagram of $B_{n}$ without the last element, then

$$
W_{\Gamma}(q, t)=[m+1]_{q} !
$$

where $m=|\Gamma|$. If $\bar{\Gamma}$ is connected and contains the last element of $B_{n}$, then

$$
W_{\Gamma}(q, t)=[2 m]_{q, t} ! !
$$

where $m=|\Gamma|$.

If $\bar{\Gamma}$ is the union of several connected components of the Dynkin diagram, $\bar{\Gamma}=$ $\bar{\Gamma}_{1} \cup \cdots \cup \bar{\Gamma}_{k}$, then $W_{\Gamma}(q, t)$ is the product

$$
\prod_{i=1}^{k} W_{\Gamma_{i}}(q, t)
$$

of the factors corresponding to the different components.

If $j \notin \Gamma$ we can write $\bar{\Gamma}(j)$ for the connected component of $\overline{\Gamma \cup\{j\}}$ containing $j$. Suppose that $m=|\Gamma(j)|$ and $i$ is the number of elements in $\Gamma(j)$ greater than $j$. Then, if $n \in \Gamma(j)$, we have

$$
\frac{W_{\Gamma \cup\{j\}}(q, t)}{W_{\Gamma}(q, t)}=\left[\begin{array}{c}
m \\
i
\end{array}\right]_{q, t}^{\prime}
$$

and

$$
\frac{W_{\Gamma \cup\{j\}}(q, t)}{W_{\Gamma}(q, t)}=\left[\begin{array}{c}
m+1 \\
i+1
\end{array}\right]_{q}
$$

otherwise.

It is convenient to represent generators $\Gamma \subset I_{n}$ by their characteristic functions $I_{n} \rightarrow\{0,1\}$ and so, simply by strings of 0 s and 1 s of length $n$.

We define a decreasing filtration $F$ on the complex $\left(C_{n}^{*}, \delta\right): F^{s} C_{n}$ is the subcomplex generated by the strings of type $A 1^{s}$ (ending with a string of $s 1 \mathrm{~s}$ ) and we have the inclusions

$$
C_{n}=F^{0} C_{n} \supset F^{1} C_{n} \supset \cdots \supset F^{n} C_{n}=R .1^{n} \supset F^{n+1} C_{n}=0 .
$$

We have the following isomorphism of complexes:

$$
\left(F^{s} C_{n} / F^{s+1} C_{n}\right) \simeq \bar{C}_{n-s-1}[s]
$$

where $\bar{C}_{n-s-1}$ is the complex for $G_{A_{n-s-1}}$ and the notation $[s]$ means that the degree is shifted by $s$. Let $E_{*}$ be the spectral sequence associated with the filtration $F$. The equality (3.2) tells us what the $E_{1}$-term of the spectral sequence looks like. In fact for $0 \leq s \leq n-2$ we have

$$
E_{1}^{s, r}=H^{r}\left(G_{A_{n-s-1}}, R_{q, t}\right)=H^{r}\left(G_{A_{n-s-1}}, \mathbb{Q}\left[q^{ \pm 1}\right]_{q}\right)\left[t^{ \pm 1}\right]
$$

since the $t$-action is trivial. For $s=n-1$ and $s=n$ the only non-trivial elements in the spectral sequence are

$$
E_{1}^{n-1,0}=E_{1}^{n, 0}=R .
$$

In order to prove Theorem 1.1 we need to state the following lemmas.

Lemma 3.1. Let $I(n, k)$ be the ideal generated by the polynomials

$$
\left[\begin{array}{c}
n \\
n-d
\end{array}\right]_{q, t}^{\prime} \quad \text { for } d \mid n \text { and } d \leq k .
$$


If $k \mid n$, the map

$$
\alpha_{n, k}: R /\left(\varphi_{k}(q)\right) \rightarrow R / I(n, k-1)
$$

induced by the multiplication by $\left[\begin{array}{c}n \\ n-k\end{array}\right]_{q, t}^{\prime}$ is well defined and is injective.

Remark. The fact that this map is well defined will follow automatically from the general theory of spectral sequences, as it is clear from the proof of Theorem 1.1 However, below we prove it by other means.

Proof. Let $d, k$ be positive integers such that $d \mid n$ and $k \mid n$. We can observe that $\varphi_{d}(q) \mid\left[\begin{array}{l}n \\ k\end{array}\right]_{q}=\left[\begin{array}{c}n \\ n-k\end{array}\right]_{q}$ if and only if $d \nmid k$. Moreover each factor $\varphi_{d}$ appears in $\left[\begin{array}{l}n \\ k\end{array}\right]_{q}$ at most with exponent 1 .

Let $J(n, k)$ be the ideal generated by the polynomials $\left[\begin{array}{c}n \\ n-d\end{array}\right]_{q}$ for $d \mid n$ and $d \leq k$. It is easy to see that we have the following inclusion:

$$
\prod_{i=n-k}^{n-1}\left(1+t q^{i}\right) J(n, k) \subset I(n, k) .
$$

Moreover $J(n, k)$ is a principal ideal and is generated by the product

$$
p_{n, k}(q)=\prod_{d \mid n, k<d} \varphi_{d}(q) .
$$

It follows that $\left[\begin{array}{c}n \\ n-k\end{array}\right]_{q} \varphi_{k}(q) \in J(n, k-1)$ and so $\left[\begin{array}{c}n \\ n-k\end{array}\right]_{q, t}^{\prime} \varphi_{k}(q) \in I(n, k-1)$. This proves that the map $\alpha_{n, k}$ is well defined.

Now we notice that the factor $\varphi_{k}(q)$ divides each generator of $I(n, k-1)$, but does not divide $\left[\begin{array}{c}n \\ n-k\end{array}\right]_{q, t}^{\prime}$. This implies that $\alpha_{n, k}$ is not the zero map and that every polynomial in the kernel of $\alpha_{n, k}$ must be a multiple of $\varphi_{k}(q)$; hence the map must be injective.

Lemma 3.2. Let $I(n)$ be the ideal generated by the polynomials

$$
\left[\begin{array}{c}
n \\
n-d
\end{array}\right]_{q, t}^{\prime} \quad \text { for } d \mid n \text {. }
$$

Then $I(n)$ is the direct product of the ideals $I_{i, d}=\left(\varphi_{d}(q), q^{i} t+1\right)$ for $d \mid n$ and $0 \leq i \leq d-2$ and of the ideal $I_{n-1}=\left(q^{n-1} t+1\right)$. Moreover the ideals $I_{i, d}$ and $I_{n-1}$ are pairwise coprime.

Proof: Notice that the polynomial $\left(1+t q^{n-1}\right)$ divides each generator of the ideal $I(n)$, so we can write

$$
I(n)=\left(1+t q^{n-1}\right) \widetilde{I}(n)
$$

where $\widetilde{I}(n)$ is the ideal generated by the polynomials

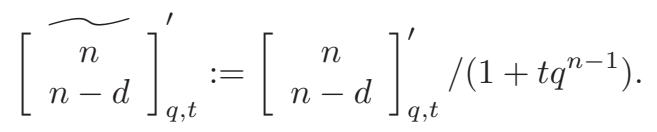


Let $n=d_{1}>\cdots>d_{h}=1$ be the list of all the divisors of $n$ in decreasing order. If we set

$$
\begin{aligned}
P_{i} & :=\varphi_{d_{i}}(q) \text { and } \\
Q_{i} & :=\prod_{j=d_{i+1}+1}^{d_{i}}\left(1+t q^{n-j}\right)
\end{aligned}
$$

we can rewrite our ideal as

$$
\begin{aligned}
\widetilde{I}(n)= & \left(\left[\begin{array}{c}
n \\
n-d_{h}
\end{array}\right],\left[\begin{array}{c}
n \\
n-d_{h-1}
\end{array}\right] Q_{h-1},\left[\begin{array}{c}
n \\
n-d_{h-2}
\end{array}\right] Q_{h-2} Q_{h-1}, \ldots\right. \\
& \left.\ldots,\left[\begin{array}{c}
n \\
n-d_{2}
\end{array}\right] Q_{2} \cdots Q_{h-1}, Q_{1} \cdots Q_{h-1}\right) .
\end{aligned}
$$

We claim that we can reduce to the following set of generators:

$$
\begin{aligned}
\widetilde{I}(n)= & \left(P_{1} \cdots P_{h-1}, P_{1} \cdots P_{h-2} Q_{h-1}, P_{1} \cdots P_{h-3} Q_{h-2} Q_{h-1}, \ldots\right. \\
& \left.\ldots, P_{1} Q_{2} \cdots Q_{h-1}, Q_{1} \cdots Q_{h-1}\right) .
\end{aligned}
$$

The first generator is the same in both equations and the $j$-th generator in (3.6) divides the corresponding generator in (3.5). Now suppose that a factor $\varphi_{m}(q)$ divides $\left[\begin{array}{c}n \\ n-d_{j}\end{array}\right]$ but does not divide $P_{1} \cdots P_{j-1}$. We may distinguish two cases:

i) Suppose that $m \nmid n$. Then we can get rid of the factor $\varphi_{m}(q)$ in $\left[\begin{array}{c}n \\ n-d_{j}\end{array}\right]$ with an opportune combination with the polynomial

$$
P_{1} \cdots P_{h-1} \text {. }
$$

ii) Suppose $m \mid n$. Then $m=d_{l}$ for some $l>j$ and we can get rid of $\varphi_{m}(q)$ using a suitable combination with the polynomial

$$
P_{1} \cdots P_{l-1} Q_{l} \cdots Q_{h-1}
$$

We may now proceed inductively. Supposing we have already reduced the first $j-1$ terms, we can reduce the $j$-th term of the ideal in (3.5) to the corresponding term in (3.6).

Now we observe that if $J, I_{1}, I_{2}$ are ideals and $I_{1}+I_{2}=(1)$, then $\left(J, I_{1} I_{2}\right)=$ $\left(J, I_{1}\right)\left(J, I_{2}\right)$. Since the polynomials $P_{i}$ are all coprime, we can apply this fact to the ideal $\widetilde{I}(n) h-2$ times. At the $i$-th step we set

$$
\begin{gathered}
I_{1}=\left(P_{i}\right) \\
I_{2}=\left(P_{i+1} \cdots P_{h-1}, P_{i+1} \cdots P_{h-2} Q_{h-1}, \ldots, Q_{i+1} \cdots Q_{h-1}\right), \\
J=\left(Q_{i} \cdots Q_{h-1}\right) .
\end{gathered}
$$

So we can factor $\widetilde{I}(n)$ as

$$
\begin{gathered}
\left(P_{1}, Q_{1} \cdots Q_{h-1}\right)\left(P_{2} \cdots P_{h-1}, P_{2} \cdots P_{h-2} Q_{h-1}, Q_{2} \cdots Q_{h-1}\right)=\cdots \\
=\left(P_{1}, Q_{1} \cdots Q_{h-1}\right)\left(P_{2}, Q_{2} \cdots Q_{h-1}\right) \cdots\left(P_{h-1}, Q_{h-1}\right) .
\end{gathered}
$$

Finally we can split $\left(P_{s}, Q_{s} \cdots Q_{h-1}\right)$ as the product

$$
\left(P_{s}, 1+t q^{n-d_{s}}\right) \cdots\left(P_{s}, 1+t q^{n-d_{h}-1}\right) .
$$

So we have reduced the ideal $I(n)$ in the product stated in the lemma, and it is easy to check that all the ideals of the splitting are coprime. 
Proof of Theorem [1.1. We can now prove our theorem using the spectral sequence described in (3.3) and (3.4).

We introduce, as in DCPS01, the following notation for the generators of the spectral sequence:

$$
\begin{aligned}
w_{h} & =01^{h-2} 0, \\
z_{h} & =1^{h-1} 0+(-1)^{h} 01^{h-1}, \\
b_{h} & =01^{h-2} \\
c_{h} & =1^{h-1} \\
z_{h}(i) & =\sum_{j=0}^{i-1}(-1)^{h j} w_{h}^{j} z_{h} w_{h}^{i-j-1}, \\
v_{h}(i) & =\sum_{j=0}^{i-2}(-1)^{h j} w_{h}^{j} z_{h} w_{h}^{i-j-2} b_{h}+(-1)^{h(i-1)} w_{h}^{i-1} c_{h} .
\end{aligned}
$$

We write $\{m\}\left[t^{ \pm 1}\right]$ for the module $R /\left(\varphi_{m}(q)\right)$. The $E_{1}$-term of the spectral sequence has a module $\{m\}\left[t^{ \pm 1}\right]$ in position $(s, r)$ if and only if one of the following conditions is satisfied:

a) $m \mid n-s-1$ and $r=n-s-2 \frac{n-s-1}{m}$;

b) $m \mid n-s$ and $r=n-s+1-2\left(\frac{n-s}{m}\right)$.

Moreover we have modules $R$ in positions $(n-1,0)$ and $(n, 0)$. We now look at the $d_{1}$ map between these two modules. Notice that $E_{1}^{n-1,0}$ is generated by the string $01^{n-1}$ and $E_{1}^{n, 0}$ is generated by the string $1^{n}$. Furthermore the map

$$
d_{1}^{n-1,0}: E_{1}^{n-1,0} \rightarrow E_{1}^{n, 0}
$$

is given by the multiplication by $\left[\begin{array}{c}n \\ n-1\end{array}\right]_{q, t}^{\prime}=[n]_{q}\left(1+t q^{n-1}\right)$ and is injective. It turns out that $E_{2}^{n-1,0}=0$ and $E_{2}^{n, 0}=R /\left([n]_{q}\left(1+t q^{n-1}\right)\right)$. Moreover all the following terms $E_{j}^{n, 0}$ are quotients of $E_{2}^{n, 0}$.

Notice that every map between modules of types $\{m\}\left[t^{ \pm 1}\right]$ and $\left\{m^{\prime}\right\}\left[t^{ \pm 1}\right]$ must be zero if $m \neq m^{\prime}$. So we can study our spectral sequence considering only maps between the same kinds of modules.

First let us consider an integer $m$ that doesn't divide $n$. Say that $m \mid n+c$ with $1 \leq c<m$ and set $i=\frac{n+c}{m}$. The modules of type $\{m\}\left[t^{ \pm 1}\right]$ are:

$$
\begin{array}{ll}
E_{1}^{\lambda m-c-1, n+c-\lambda(m-2)-2 i+1} & \text { generated by } z_{m}(i-\lambda) 01^{\lambda m-c-1}, \\
E_{1}^{\lambda m-c, n+c-\lambda(m-2)-2 i+1} & \text { generated by } v_{m}(i-\lambda) 01^{\lambda m-c}
\end{array}
$$

for $\lambda=1, \ldots, i-1$.

Here is a diagram for this case (we use the notation $\boldsymbol{h}$ for $\{m\}\left[t^{ \pm 1}\right]$ ):

$$
h \stackrel{d_{1}}{\rightarrow} h
$$

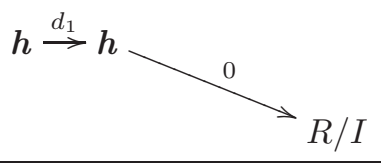


The map

$$
d_{1}: E_{1}^{\lambda m-c-1, n+c-\lambda(m-2)-2 i+1} \rightarrow E_{1}^{\lambda m-c, n+c-\lambda(m-2)-2 i+1}
$$

is given by the multiplication by $\left[\begin{array}{c}\lambda m-c \\ \lambda m-c-1\end{array}\right]_{q, t}^{\prime}=[\lambda m-c]_{q}\left(1+t q^{\lambda m-c-1}\right)$. Since $\varphi_{m}(q) \nmid[\lambda m-c]_{q}$ the map is injective and in the $E_{2}$-term we have:

$$
\begin{array}{ll}
E_{2}^{\lambda m-c-1, n+c-\lambda(m-2)-2 i+1} & =0, \\
E_{2}^{\lambda m-c, n+c-\lambda(m-2)-2 i+1} & =\{m\}_{\lambda m-c-1}=\{m\}_{m-c-1}
\end{array}
$$

for $\lambda=1, \ldots, i-1$.

The other map we have to consider is

$$
d_{m}^{n-m, m-1}: E_{m}^{n-m, m-1} \rightarrow E_{m}^{n, 0} .
$$

The module $E_{m}^{n-m, m-1}=\{m\}_{m-c-1}$ is generated by $1^{m-1} 01^{n-m}$ and so the map is the multiplication by $\left[\begin{array}{c}n \\ n-m\end{array}\right]_{q, t}^{\prime}$. Since $\left(1+t q^{n-1}\right)$ divides the coefficient $\left[\begin{array}{c}n \\ n-m\end{array}\right]_{q, t}^{\prime}$, the image of the map $d_{m}^{n-m, m-1}$ must be contained in the submodule

$$
\left(1+t q^{n-1}\right) E_{m}^{n, 0}=\left(1+t q^{n-1}\right) R /\left([n]_{q}\left(1+t q^{n-1}\right)\right),
$$

that is, in the quotient $R /\left([n]_{q}\right)$. Since $\left(\varphi_{m}(q),[n]_{q}\right)=(1)$ (recall that $m$ does not divide $n$ ) there can be no non-trivial map between the modules $\{m\}_{m-c-1}$ and $R /\left([n]_{q}\right)$. It follows that the differential $d_{m}^{n-m, m-1}$ must be zero.

As a consequence the $E_{2}$ part described before collapses to $E_{\infty}$ and we have a copy of $\{m\}_{m-c-1}$ as a direct summand of $H^{n-2 j-1}\left(C_{n}\right)$ for $j=0, \ldots, i-2$, that is, for $m \leq \frac{n}{j+1}$.

Now we consider an integer $m$ that divides $n$ and let $i=\frac{n}{m}$. The modules of type $\{m\}\left[t^{ \pm 1}\right]$ are:

$$
\begin{array}{lll}
E_{1}^{\lambda m-1, n-\lambda(m-2)-2 i+1} & \text { generated by } & z_{m}(i-\lambda) 01^{\lambda m-1} \text { for } 1 \leq \lambda \leq i-1, \\
E_{1}^{\lambda m, n-\lambda(m-2)-2 i+1} & \text { generated by } & v_{m}(i-\lambda) 01^{\lambda m} \text { for } 0 \leq \lambda \leq i-1 .
\end{array}
$$

The situation is shown in the next diagram $\left(\boldsymbol{h}=\{m\}\left[t^{ \pm 1}\right]\right)$ :

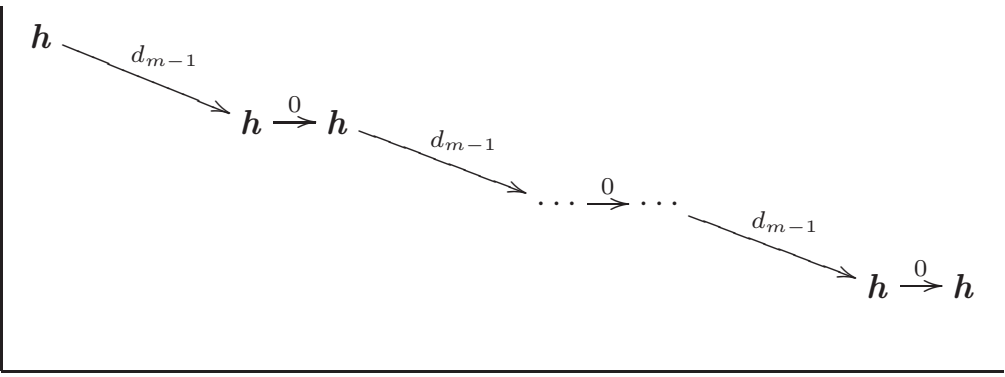

The map

$$
d_{1}: E_{1}^{\lambda m-1, n-\lambda(m-2)-2 i+1} \rightarrow E_{1}^{\lambda m, n-\lambda(m-2)-2 i+1}
$$

is given by the multiplication by $\left[\begin{array}{c}\lambda m \\ \lambda m-1\end{array}\right]_{q, t}^{\prime}=[\lambda m]_{q}\left(1+t q^{\lambda m-1}\right)$, but in this case the coefficient is zero in the module $\{m\}\left[t^{ \pm 1}\right]$ because $\varphi_{m}(q) \mid[\lambda m]_{q}$ and so 
we have that $E_{1}=\cdots=E_{m-1}$. So we have to consider the map

$$
d_{m-1}^{\lambda m, n-\lambda(m-2)-2 i+1}: E_{m-1}^{\lambda m, n-\lambda(m-2)-2 i+1} \rightarrow E_{1}^{(\lambda+1) m-1, n-(\lambda+1)(m-2)-2 i+1}
$$

for $\lambda=0, \ldots, i-2$.

This map corresponds to multiplication by

$$
\left[\begin{array}{c}
(\lambda+1) m-1 \\
\lambda m
\end{array}\right]_{q, t}^{\prime}=\left[\begin{array}{c}
(\lambda+1) m-1 \\
\lambda m
\end{array}\right]_{q} \prod_{j=\lambda m+1}^{(\lambda+1) m-1}\left(1+t q^{j-1}\right) .
$$

It is easy to see that the polynomial $\left[\begin{array}{c}(\lambda+1) m-1 \\ \lambda m\end{array}\right]_{q}$ is prime with the torsion $\varphi_{m}(q)$ and so the map $d_{m-1}^{\lambda m, n-\lambda(m-2)-2 i+1}$ is injective and the cokernel is isomorphic to

$$
R /\left(\varphi_{m}(q), \prod_{j=\lambda m+1}^{(\lambda+1) m-1}\left(1+t q^{j-1}\right)\right) \simeq \bigoplus_{0 \leq k \leq m-2}\{m\}_{k} .
$$

As a consequence we have that

$$
\begin{aligned}
& E_{m}^{\lambda m-1, n-\lambda(m-2)-2 i+1}=\bigoplus_{0 \leq k \leq m-2}\{m\}_{k} \quad \text { for } 1 \leq \lambda \leq i-1, \\
& E_{m}^{\lambda m, n-\lambda(m-2)-2 i+1}=0 \quad \text { for } 0 \leq \lambda \leq i-2 \text {, }
\end{aligned}
$$

and all these modules collapse to $E_{\infty}$. This means that we can find $\varphi_{m}(q)$-torsion only in $H^{n-2 j}\left(C_{n}\right)$ and for $j \geq 1$ the summand is given by

$$
\bigoplus_{0 \leq k \leq m-2}\{m\}_{k}
$$

for $d \leq \frac{n}{j+1}$.

We still have to consider all the terms $E_{m}^{n-m, m-1}=\{m\}\left[t^{ \pm 1}\right]$ for $m \mid n$. Here the maps we have to look at are the following:

$$
d_{m}^{n-m, m-1}: E_{m}^{n-m, m-1} \rightarrow E_{m}^{n, 0} .
$$

These maps correspond to multiplication by the polynomials $\left[\begin{array}{c}n \\ n-m\end{array}\right]_{q, t}^{\prime}$. Moreover recall that

$$
E_{1}^{n, 0}=R /\left(\left[\begin{array}{c}
n \\
n-1
\end{array}\right]_{q, t}^{\prime}\right)
$$

We can now use Lemma 3.1 to say that all the maps $d_{m}^{n-m, m-1}$ are injective and Lemma 3.2 to say that

$$
E_{n+1}^{n, 0}=E_{\infty}^{n, 0}=\bigoplus_{m \mid n, 0 \leq k \leq d-2}\{m\}_{k} \oplus\{1\}_{n-1} .
$$

Since $E_{\infty}^{n, 0}=H^{n}\left(C_{n}\right)$, this complete the proof of the theorem.

3.2. Other computations. We may also consider the cohomology of $G_{B_{n}}$ over the module $\mathbb{Q}\left[t^{ \pm 1}\right]$, where the action is trivial for the generators $\epsilon_{1}, \ldots, \epsilon_{n-1}$ and $(-t)$-multiplication for the last generator $\bar{\epsilon}_{n}$. This cohomology is computed by the complex $C_{n}^{*}$ of Section 3 where we specialize $q$ to -1 . So we may use a similar filtration and associated spectral sequence. We used this argument in CMS]. Here we 
briefly indicate a different and more concise method, using the results of Theorem 1.1. We have:

Theorem 3.3.

$$
\begin{array}{ll}
H^{k}\left(G_{B_{n}}, \mathbb{Q}\left[t^{ \pm 1}\right]\right)=\mathbb{Q}\left[t^{ \pm 1}\right] /(1+t) & 1 \leq k \leq n-1, \\
H^{n}\left(G_{B_{n}}, \mathbb{Q}\left[t^{ \pm 1}\right]\right)=\mathbb{Q}\left[t^{ \pm 1}\right] /(1+t) & \text { for odd } n, \\
H^{n}\left(G_{B_{n}}, \mathbb{Q}\left[t^{ \pm 1}\right]\right)=\mathbb{Q}\left[t^{ \pm 1}\right] /\left(1-t^{2}\right) & \text { for even } n .
\end{array}
$$

Sketch of proof. Consider the short exact sequence:

$$
0 \rightarrow \mathbb{Q}\left[q^{ \pm 1}, t^{ \pm 1}\right] \stackrel{1+q}{\longrightarrow} \mathbb{Q}\left[q^{ \pm 1}, t^{ \pm 1}\right] \rightarrow \mathbb{Q}\left[t^{ \pm 1}\right] \rightarrow 0
$$

and the induced long exact sequence for cohomology

$$
\cdots \rightarrow H^{i}\left(G_{B_{n}}, \mathbb{Q}\left[q^{ \pm 1}, t^{ \pm 1}\right]\right) \stackrel{1+q}{\longrightarrow} H^{i}\left(G_{B_{n}}, \mathbb{Q}\left[q^{ \pm 1}, t^{ \pm 1}\right]\right) \rightarrow H^{i}\left(G_{B_{n}}, \mathbb{Q}\left[t^{ \pm 1}\right]\right) \rightarrow \cdots .
$$

The result is now a straightforward consequence of Theorem 1.1 .

\section{More Consequences}

By means of Shapiro's lemma (see for instance Bro82]), the inclusions introduced in Section 2.2 can be exploited to link the cohomology of the Artin group of type $\tilde{A}_{n-1}, A_{n}$ to the cohomology of $G_{B_{n}}$.

4.1. Cohomology of $G_{\tilde{A}_{n-1}}$. Let $M$ be any domain and let $q$ be a unit of $M$. We indicate by $M_{q}$ the $\operatorname{ring} M$ with the $G_{\tilde{A}_{n-1}}$-module structure where the action of the standard generators is given by $(-q)$-multiplication.

Proposition 4.1. We have

$$
\begin{aligned}
& H_{*}\left(G_{\tilde{A}_{n-1}}, M_{q}\right) \cong H_{*}\left(G_{B_{n}}, M\left[t^{ \pm 1}\right]_{q, t}\right), \\
& H^{*}\left(G_{\tilde{A}_{n-1}}, M_{q}\right) \cong H^{*}\left(G_{B_{n}}, M\left[\left[t^{ \pm 1}\right]\right]_{q, t}\right)
\end{aligned}
$$

where the action of $G_{B_{n}}$ on $M\left[t^{ \pm 1}\right]_{q, t}$ (and on $M\left[\left[t^{ \pm 1}\right]\right]_{q, t}$ ) is given by $(-q)$-multiplication for the generators $\epsilon_{1}, \ldots, \epsilon_{n-1}$ and $(-t)$-multiplication for the last generator $\bar{\epsilon}_{n}$.

Proof. Applying Shapiro's lemma to the inclusion $\tilde{A}_{n-1}<G_{B_{n}}$, one obtains:

$$
\begin{aligned}
& H_{*}\left(G_{\tilde{A}_{n-1}}, M_{q}\right) \cong H_{*}\left(G_{B_{n}}, \operatorname{Ind}_{G_{\tilde{A}_{n-1}}}^{G_{B_{n}}} M_{q}\right), \\
& H^{*}\left(G_{\tilde{A}_{n-1}}, M_{q}\right) \cong H^{*}\left(G_{B_{n}}, \operatorname{Coind}_{G_{\tilde{A}_{n-1}}}^{G_{\tilde{A}_{n}}} M_{q}\right) .
\end{aligned}
$$

By Corollary 2.3. any element of $\operatorname{Ind}_{G_{\tilde{A}_{n-1}}}^{G_{B_{n}}} M_{q}:=\mathbb{Z}\left[G_{B_{n}}\right] \otimes_{G_{\tilde{A}_{n-1}}} M_{q}$ can be represented as a sum of elements of the form $\tau^{\alpha} \otimes q^{m}$. Now, we have an isomorphism of $\mathbb{Z}\left[G_{B_{n}}\right]$-modules

$$
\mathbb{Z}\left[G_{B_{n}}\right] \otimes_{G_{\tilde{A}_{n-1}}} M_{q} \rightarrow M\left[t^{ \pm 1}\right]_{q, t}
$$

defined by sending $\tau^{\alpha} \otimes q^{m} \mapsto(-1)^{n \alpha} t^{\alpha} q^{(n-1) \alpha+m}$ and the result follows.

In cohomology we have similarly:

$$
\operatorname{Coind}_{G_{\tilde{A}_{n-1}}}^{G_{B_{n}}} M_{q}:=\operatorname{Hom}_{G_{\tilde{A}_{n-1}}}\left(\mathbb{Z}\left[G_{B_{n}}\right], M_{q}\right) \cong M\left[\left[t^{ \pm 1}\right]\right]_{q, t} .
$$


By Propositions 4.1, in order to determine the cohomology $H^{*}\left(G_{\tilde{A}_{n-1}}, M_{q}\right)$ it is necessary to know the cohomology of $G_{B_{n}}$ with values in the module $M\left[\left[t^{ \pm 1}\right]\right]$ of Laurent series in the variable $t$. The latter is linked to the cohomology with values in the module of Laurent polynomials by:

Proposition 4.2 (Degree shift).

$$
H^{*}\left(G_{B_{n}}, M\left[\left[t^{ \pm 1}\right]\right]_{q, t}\right) \cong H^{*+1}\left(G_{B_{n}}, M\left[t^{ \pm 1}\right]_{q, t}\right) .
$$

This result was obtained in Cal05 in a slightly weaker form, but it is possible to extend it to our case with little effort.

From now on let $M=\mathbb{Q}\left[q^{ \pm 1}\right]$. In this case we have $M\left[t^{ \pm 1}\right]_{q, t}=R_{q, t}$, so we obtain the cohomology of the Artin group of affine type $\tilde{A}_{n-1}$ with $M_{q}$-coefficients by means of Theorem 1.1 .

In a similar way we get the rational cohomology of $G_{\tilde{A}_{n-1}}$ :

Proposition 4.3. We have

$$
\begin{aligned}
& H_{*}\left(G_{\tilde{A}_{n-1}}, \mathbb{Q}\right) \cong H_{*}\left(G_{B_{n}}, \mathbb{Q}\left[t^{ \pm 1}\right]\right), \\
& H^{*}\left(G_{\tilde{A}_{n-1}}, \mathbb{Q}\right) \cong H^{*}\left(G_{B_{n}}, \mathbb{Q}\left[\left[t^{ \pm 1}\right]\right]\right)
\end{aligned}
$$

where the action of $G_{B_{n}}$ on $\mathbb{Q}\left[t^{ \pm 1}\right]$ (and on $\mathbb{Q}\left[\left[t^{ \pm 1}\right]\right]$ ) is trivial for the generators $\epsilon_{1}, \ldots, \epsilon_{n-1}$ and $(-t)$-multiplication for the last generator $\bar{\epsilon}_{n}$.

To obtain the rational cohomology of $G_{\tilde{A}_{n-1}}$ we may apply Proposition 4.2 together with Theorem 3.3 .

4.2. Cohomology of $G_{A_{n}}$ with coefficient in the Tong-Yang-Ma representation. The Tong-Yang-Ma representation is an $(n+1)$-dimensional representation of the classical braid group $G_{A_{n}}$ discovered in [TYM96]. Below we just recall it, referring to Sys01 for a discussion of its relevance in braid group representation theory.

Definition 4.4. Let $V$ be the free $\mathbb{Q}\left[u^{ \pm 1}\right]$-module of rank $n+1$. The Tong-Yang-Ma representation is the representation

$$
\rho: G_{A_{n}} \rightarrow \mathrm{Gl}_{\mathbb{Q}\left[u^{ \pm 1}\right]}(V)
$$

defined w.r.t. the basis $e_{1}, \ldots, e_{n+1}$ of $V$ by:

$$
\rho\left(\sigma_{i}\right)=\left(\begin{array}{cccc}
I_{i-1} & & & \\
& 0 & 1 & \\
& u & 0 & \\
& & & I_{n-i}
\end{array}\right)
$$

where $I_{j}$ denotes the $j$-dimensional identity matrix and all other entries are zero.

Notice that the image of the pure braid group under the Tong-Yang-Ma representation is abelian; hence this representation factors through the extended Coxeter group presented in Tit66].

Proposition 4.5. We have

$$
\begin{aligned}
& H_{*}\left(G_{B_{n}}, M\left[t^{ \pm 1}\right]_{q, t}\right) \cong H_{*}\left(G_{A_{n}}, M_{q} \otimes V\right), \\
& H^{*}\left(G_{B_{n}}, M\left[t^{ \pm 1}\right]_{q, t}\right) \cong H^{*}\left(G_{A_{n}}, M_{q} \otimes V\right)
\end{aligned}
$$

where each generator of $G_{A_{n}}$ acts on $M_{q} \otimes V$ by $(-q)$-multiplication on the first factor and by the Tong-Yang-Ma representation on the second factor. 
Sketch of proof. For the statement in homology, by Shapiro's lemma, it is enough to show that $\operatorname{Ind}_{G_{B_{n}}}^{G_{A_{n}}} M\left[t^{ \pm 1}\right]_{q, t} \cong M_{q} \otimes V$.

Notice that $\left[G_{A_{n}}: G_{B_{n}}\right]=n+1$ and let us choose as coset representatives for $G_{A_{n}} / G_{B_{n}}$ the elements $\alpha_{i}=\left(\sigma_{i} \sigma_{i+1} \cdots \sigma_{n-1}\right) \sigma_{n}\left(\sigma_{i} \sigma_{i+1} \cdots \sigma_{n-1}\right)^{-1}$ for $1 \leq i \leq$ $n-1, \alpha_{n}=\sigma_{n}, \alpha_{n+1}=e$.

Then by the definition of induced representation, there is an isomorphism of left $G_{A_{n}}$-modules,

$$
\operatorname{Ind}_{G_{B_{n}}}^{G_{A_{n}}} M\left[t^{ \pm 1}\right]_{q, t}=\bigoplus_{i=1}^{n+1} M\left[t^{ \pm 1}\right] e_{i}
$$

where the action on the r.h.s. is as follows. For an element $x \in G_{A_{n}}$, write $x \alpha_{k}=\alpha_{k^{\prime}} x^{\prime}$ with $x^{\prime} \in G_{B_{n}}$. Then $x$ acts on an element $r \cdot e_{k} \in \bigoplus_{i=1}^{n+1} M\left[t^{ \pm 1}\right] e_{i}$ as $x\left(r \cdot e_{k}\right)=\left(x^{\prime} r\right) \cdot e_{k^{\prime}}$.

Computing explicitly this action for the standard generators of $G_{A_{n}}$, we can write the representation in the following matrix form:

$$
\sigma_{i} \mapsto\left(\begin{array}{cccc}
-q I_{i-1} & & & \\
& 0 & -q & \\
& q^{-1} t & 0 & \\
& & & -q I_{n-i}
\end{array}\right)
$$

for $1 \leq i \leq n-1$, whereas

$$
\sigma_{n} \mapsto\left(\begin{array}{ccc}
-q I_{n-1} & & \\
& 0 & 1 \\
& -t & 0
\end{array}\right) .
$$

Conjugating by $U=\operatorname{Diag}\left(1,1, \ldots, 1,-q^{-1}\right)$ and setting $u=-q^{-2} t$, one obtains the desired result.

Finally, since $\left[G_{A_{n}}: G_{B_{n}}\right]=n+1<\infty$, the induced and coinduced representations are isomorphic; so the analogous statement in cohomology follows.

In particular the cohomology of $G_{B_{n}}$ determined in Theorem 1.1 is isomorphic to the cohomology of $G_{A_{n}}$ with coefficient in the Tong-Yang-Ma representation twisted by an abelian representation.

By means of Shapiro's lemma, we may as well determine the cohomology of $G_{A_{n}}$ with coefficient in the Tong-Yang-Ma representation. Indeed:

Proposition 4.6. We have

$$
\begin{aligned}
& H_{*}\left(G_{B_{n}}, \mathbb{Q}\left[t^{ \pm 1}\right]\right) \cong H_{*}\left(G_{A_{n}}, V\right), \\
& H^{*}\left(G_{B_{n}}, \mathbb{Q}\left[t^{ \pm 1}\right]\right) \cong H^{*}\left(G_{A_{n}}, V\right)
\end{aligned}
$$

where $V$ is the representation of $G_{A_{n}}$ defined in Definition 4.4 .

As a consequence we have

Corollary 4.7. Let $V$ be the $(n+1)$-dimensional representation of the braid group $\mathrm{Br}_{n+1}$ defined in Definition 4.4. Then the cohomology

$$
H^{*}\left(\mathrm{Br}_{n+1}, V\right)
$$

is given as in Theorem 3.3

Remark 4.8. In particular the homology of $G_{\tilde{A}_{n-1}}$ with trivial coefficients is isomorphic to the homology of $G_{A_{n}}$ with coefficients in the Tong-Yang-Ma representation. 


\section{RELATED topological CONSTRUCtions}

We refer to [CMS for the few changes which have to be done to the construction given in Sal94] (see also Sal87]) for non-finite type Artin groups (but still finitely generated). We obtain a finite $\mathrm{CW}$-complex $X_{W}$, explicitly described, which is a deformation retract of the orbit space of the Artin group. The latter is defined as the quotient space

$$
\mathbf{M}(\mathcal{A})_{W}:=\mathbf{M}(\mathcal{A}) / W
$$

where

$$
\mathbf{M}(\mathcal{A}):=\left[U^{0}+i \mathbb{R}^{n}\right] \backslash \bigcup_{H \in \mathcal{A}} H_{\mathbb{C}}
$$

$U^{0} \subset \mathbb{R}^{n}$ being the interior part of the Tits cone of $W$, while $\mathcal{A}$ is the hyperplane arrangement of $W$. The associated Artin group $G_{W}$ is the fundamental group of the orbit space (see [Bou68, Vin71, Bri71, Dũn83, vdL83]).

The simplest way to realize $X_{W}$ is by taking one point $x_{0}$ inside a chamber $C_{0}$ and, for any maximal subset $J \subset S$ such that the parabolic subgroup $W_{J}$ is finite, construct a $|J|$-cell (a polyhedron) in $U^{0}$ as the "convex hull" of the $W_{J}$-orbit of $x_{0}$ in $\mathbb{R}^{n}$. So, we obtain a finite cell complex which is the union of (in general, different dimensional) polyhedra. Next, there are identifications on the faces of these polyhedra, which are the same as described in Sal94 for the finite case. The resulting quotient space is a CW-complex $X_{W}$ which has a $|J|$-cell for each $J \subset S$ such that $W_{J}$ is finite. We show an example in the case $\tilde{A}_{2}$ (see Figure 4 ).

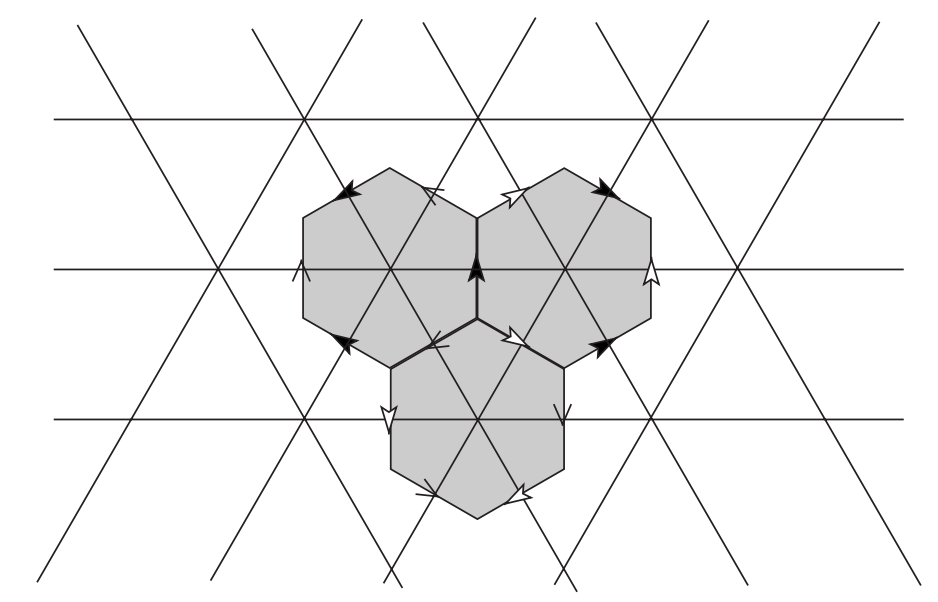

FIGURE 4. The space $K\left(G_{\tilde{A}_{2}}, 1\right)$ is given as a union of 3 hexagons with edges glued according to the arrows (there are: 10 -cell, 3 1-cells, 32 -cells in the quotient).

Remark 5.1. When $W$ is an affine group, the orbit space is known to be a $K(\pi, 1)$ for types $\tilde{A}_{n}, \tilde{C}_{n}$ (see Oko79, CP03]) and recently for type $\tilde{B}_{n}$ (CMS1]); see CD95. for further classes.

Remark 5.2. The standard presentation for $G_{W}$ is quite easy to derive from the topological description of $X_{W}$; we may thus recover van der Lek's result vdL83. 
It follows that

Proposition 5.3. Let $K_{W}^{f i n}:=\left\{J \subset S:\left|W_{J}\right|<\infty\right\}$ with the natural structure of a simplicial complex. Then the Euler characteristic of the orbit space (so, of the group $G_{W}$ when such a space is of type $K(\pi, 1)$ ) equals

$$
\chi\left(K_{W}^{f i n}\right) .
$$

If $W$ is affine of rank $n+1$ we have

$$
\chi\left(\mathbf{M}(\mathcal{A})_{W}\right)=\chi\left(K_{W}^{f i n}\right)=1-\chi\left(S^{n-1}\right)=(-1)^{n} .
$$

If $W$ is two-dimensional (so, all 3-subsets of $S$ generate an infinite group) of rank $n$, then

$$
\chi\left(\mathbf{M}(\mathcal{A})_{W}\right)=1-n+m
$$

where $m$ is the number of pairs in $S$ having finite weight $\left(m=\frac{n(n-1)}{2}\right.$ if there are no $\infty$-edges in the Coxeter graph).

Proof. The first two statements were already mentioned in [CMS. The last one is clear.

Remark 5.4. The cohomology of the orbit space in case $\tilde{A}_{n}$ with trivial coefficients is deduced from Corollary 4.3 and from Theorem 3.3, that with local coefficients in the $G_{\tilde{A}_{n}}$-module $\mathbb{Q}\left[q^{ \pm 1}\right]$ is deduced from Theorem 1.1

\section{REFERENCES}

[All02] D. Allcock, Braid pictures for Artin groups, Trans. Amer. Math. Soc. 354 (2002), no. 9, 3455-3474 (electronic). MR 1911508 (2003f:20053)

[Arn68] V. I. Arnol'd, Braids of algebraic functions and cohomologies of swallowtails, Uspehi Mat. Nauk 23 (1968), no. 4 (142), 247-248. MR0231828 (38:156)

[Bou68] N. Bourbaki, Éléments de mathématique. Fasc. XXXIV. Groupes et algèbres de Lie. Chapitre IV: Groupes de Coxeter et systèmes de Tits. Chapitre V: Groupes engendrés par des réflexions. Chapitre VI: systèmes de racines, Actualités Scientifiques et Industrielles, No. 1337, Hermann, Paris, 1968. MR 0240238 (39:1590)

[Bri71] E. Brieskorn, Die Fundamentalgruppe des Raumes der regulären Orbits einer endlichen komplexen Spiegelungsgruppe, Invent. Math. 12 (1971), 57-61. MR0293615 $(45: 2692)$

[Bro82] K. S. Brown, Cohomology of groups, Graduate Texts in Mathematics, vol. 87, Springer-Verlag, New York, 1982. MR672956 (83k:20002)

[BS72] E. Brieskorn and K. Saito, Artin-Gruppen und Coxeter-Gruppen, Invent. Math. 17 (1972), 245-271. MR0323910(48:2263)

[Cal05] F. Callegaro, On the cohomology of Artin groups in local systems and the associated Milnor fiber, J. Pure Appl. Algebra 197 (2005), no. 1-3, 323-332. MR2123992 (2005k:20090)

[Cal06] F. Callegaro, The homology of the Milnor fiber for classical braid groups, Algebr. Geom. Topol. 6 (2006), 1903-1923 (electronic). MR.2263054

[CD95] R. Charney and M. W. Davis, The $K(\pi, 1)$-problem for hyperplane complements associated to infinite reflection groups, J. Amer. Math. Soc. 8 (1995), no. 3, 597-627. MR.1303028(95i:52011)

[CMS] F. Callegaro, D. Moroni, and M. Salvetti, Cohomology of Artin braid groups of type $\tilde{A}_{n}, B_{n}$ and applications, To be published in Geometry \& Topology Monographs.

[CMS1] F. Callegaro, D. Moroni, and M. Salvetti, The $K(\pi, 1)$-problem for the affine Artin group of type $\tilde{B}_{n}$ and its cohomology, (2006), to appear

[Coh76] F. R. Cohen, The homology of iterated loop spaces, Lecture Notes in Mathematics, vol. 533, The homology of $C_{n+1}$-spaces, $n \geq 0$, pp. 207-351, Springer-Verlag, Berlin, 1976. MR0436146(55:9096) 
[CP03] R. Charney and D. Peifer, The $K(\pi, 1)$-conjecture for the affine braid groups, Comment. Math. Helv. 78 (2003), no. 3, 584-600. MR1998395 (2004f:20067)

[Cri99] J. Crisp, Injective maps between Artin groups, Geometric group theory down under (Canberra, 1996), de Gruyter, Berlin, 1999, pp. 119-137. MR1714842 (2001b:20064)

[CS98] D. C. Cohen and A. I. Suciu, Homology of iterated semidirect products of free groups, J. Pure Appl. Algebra 126 (1998), no. 1-3, 87-120. MR.1600518 (99e:20064)

[DCPS01] C. De Concini, C. Procesi, and M. Salvetti, Arithmetic properties of the cohomology of braid groups, Topology 40 (2001), no. 4, 739-751. MR.1851561 (2002f:20082)

[DCPSS99] C. De Concini, C. Procesi, M. Salvetti, and F. Stumbo, Arithmetic properties of the cohomology of Artin groups, Ann. Scuola Norm. Sup. Pisa Cl. Sci. (4) 28 (1999), no. 4, 695-717. MR1760537|(2001f:20078)

[DCS96] C. De Concini and M. Salvetti, Cohomology of Artin groups, Math. Res. Lett. 3 (1996), no. 2, 293-297. MR.1386847 (97b:52015)

[DCSS97] C. De Concini, M. Salvetti, and F. Stumbo, The top-cohomology of Artin groups with coefficients in rank-1 local systems over $\mathbf{Z}$, Special issue on braid groups and related topics (Jerusalem, 1995), vol. 78, Topology Appl., no. 1-2, 1997, pp. 5-20. MR $1465022(98 \mathrm{~h}: 20063)$

[Del72] P. Deligne, Les immeubles des groupes de tresses généralisés, Invent. Math. 17 (1972), 273-302. MR0422673 (54:10659)

[Dũn83] Nguyẽn Viêt Dũng, The fundamental groups of the spaces of regular orbits of the affine Weyl groups, Topology 22 (1983), no. 4, 425-435. MR715248 (85f:57001)

[Fre88] Ė. V. Frenkel', Cohomology of the commutator subgroup of the braid group, Funktsional. Anal. i Prilozhen. 22 (1988), no. 3, 91-92. MR961774 (90h:20055)

[Fuk70] D. B. Fuks, Cohomology of the braid group mod 2, Funkcional. Anal. i Priložen. 4 (1970), no. 2, 62-73. MR0274463 (43:226)

[Gor78] V. V. Gorjunov, The cohomology of braid groups of series $C$ and $D$ and certain stratifications, Funktsional. Anal. i Prilozhen. 12 (1978), no. 2, 76-77. MR498905 (80g:32020)

[Hum90] J. E. Humphreys, Reflection groups and Coxeter groups, Cambridge Studies in Advanced Mathematics, vol. 29, Cambridge University Press, Cambridge, 1990. MR.1066460(92h:20002)

[KP02] R. P. Kent, IV and D. Peifer, A geometric and algebraic description of annular braid groups, Internat. J. Algebra Comput. 12 (2002), no. 1-2, 85-97, International Conference on Geometric and Combinatorial Methods in Group Theory and Semigroup Theory (Lincoln, NE, 2000). MR 1902362 (2003f:20056)

[Lam94] S. S. F. Lambropoulou, Solid torus links and Hecke algebras of B-type, Proceedings of the Conference on Quantum Topology (Manhattan, KS, 1993), World Sci. Publ., River Edge, NJ, 1994, pp. 225-245. MR1309934 (96a:57020)

[Lan00] C. Landi, Cohomology rings of Artin groups, Atti Accad. Naz. Lincei Cl. Sci. Fis. Mat. Natur. Rend. Lincei (9) Mat. Appl. 11 (2000), no. 1, 41-65. MR1797053(2001j:20082)

[Mar96] N. S. Markaryan, Homology of braid groups with nontrivial coefficients, Mat. Zametki 59 (1996), no. 6, 846-854. MR 1445470 (98j:20047)

[Oko79] C. Okonek, Das $K(\pi, 1)$-Problem für die affinen Wurzelsysteme vom Typ $A_{n}, C_{n}$, Math. Z. 168 (1979), no. 2, 143-148. MR544701 (80i:32039)

[Rei93] V. Reiner, Signed permutation statistics, European J. Combin. 14 (1993), no. 6, 553567. MR.1248063 (95e:05008)

[Sal87] M. Salvetti, Topology of the complement of real hyperplanes in $\mathbf{C}^{N}$, Invent. Math. 88 (1987), no. 3, 603-618. MR884802 (88k:32038)

[Sal94] M. Salvetti, The homotopy type of Artin groups, Math. Res. Lett. 1 (1994), no. 5, 565-577. MR 1295551 (95j:52026)

[Squ94] C. C. Squier, The homological algebra of Artin groups, Math. Scand. 75 (1994), no. 1, 5-43. MR 1308935 (95k:20059)

[SS97] M. Salvetti and F. Stumbo, Artin groups associated to infinite Coxeter groups, Discrete Math. 163 (1997), no. 1-3, 129-138. MR1428564(98d:20043)

[Sys01] I. Sysoeva, Dimension $n$ representations of the braid group on $n$ strings, J. Algebra 243 (2001), no. 2, 518-538. MR1850645 (2002h:20054)

[Tit66] J. Tits, Normalisateurs de tores. I. Groupes de Coxeter étendus, J. Algebra 4 (1966), 96-116. MR0206117 (34:5942) 
[TYM96] Dian-Min Tong, Shan-De Yang, and Zhong-Qi Ma, A new class of representations of braid groups, Comm. Theoret. Phys. 26 (1996), no. 4, 483-486. MR.1456851 (98c:20073)

[Vaŭ78] F. V. Vaŭnšteĭn, The cohomology of braid groups, Funktsional. Anal. i Prilozhen. 12 (1978), no. 2, 72-73. MR 498903 (80g:32019)

[vdL83] H. van der Lek, The homotopy type of complex hyperplane complements, Ph.D. thesis, Katholieke Universiteit te Nijimegen, 1983.

[Vin71] È. B. Vinberg, Discrete linear groups that are generated by reflections, Izv. Akad. Nauk SSSR Ser. Mat. 35 (1971), 1072-1112. MR0302779 (46:1922)

Scuola Normale Superiore, P.za dei Cavalieri, 7, Pisa, Italy

E-mail address: f.callegaro@sns.it

Dipartimento di Matematica "G.Castelnuovo", P.za A. Moro, 2, Roma, Italy - and ISTI-CNR, Via G. Moruzzi, 3, Pisa, Italy

E-mail address: davide.moroni@isti.cnr.it

Dipartimento di Matematica "L.Tonelli", Largo B. Pontecorvo, 5, Pisa, italy

E-mail address: salvetti@dm.unipi.it 